\title{
Are Thyroid Autoimmune Diseases Associated with Cardiometabolic Risks in a Population with Normal Thyroid-Stimulating Hormone?
}

\author{
Yi Chen $\mathbb{D}^{1},{ }^{1}$ Chunfang Zhu, ${ }^{1}$ Yingchao Chen, ${ }^{1}$ Ningjian Wang $\mathbb{D},{ }^{1}$ Qin $\mathrm{Li}^{1}{ }^{1}$ Bing Han, ${ }^{1}$ \\ Li Zhao, ${ }^{1}$ Chi Chen, ${ }^{1}$ Hualing Zhai, ${ }^{1}$ Lijuan Zhang $\mathbb{D}^{2}{ }^{2}$ and Yingli Lu ${ }^{1}{ }^{1}$ \\ ${ }^{1}$ Institute and Department of Endocrinology and Metabolism, Shanghai Ninth People's Hospital, Shanghai Jiaotong University \\ School of Medicine, Shanghai, China \\ ${ }^{2}$ Department of Endocrinology, Songjiang Hospital Affiliated to the First People's Hospital of Shanghai Jiaotong University, \\ Shanghai, China
}

Correspondence should be addressed to Lijuan Zhang; 891158@163.com and Yingli Lu; luyingli2008@126.com

Received 22 April 2018; Revised 17 August 2018; Accepted 30 August 2018; Published 10 October 2018

Academic Editor: Paola Migliorini Copyright ( $) 2018$ Yi Chen et al. This is an open access article distributed under the Creative Commons Attribution License, which
permits unrestricted use, distribution, and reproduction in any medium, provided the original work is properly cited.

\begin{abstract}
Background. The interrelation between thyroid autoimmunity and cardiovascular risks is complex and has not been confirmed. This study aimed at evaluating whether there exists a relationship between thyroid autoimmune diseases (AITDs) and cardiometabolic risks in a large population with normal thyroid-stimulating hormone (TSH) levels. Methods. The data was obtained from a cross-sectional study (SPECT-China study). This study enrolled 9082 subjects (3948 males and 5134 females) above 18 years with normal TSH levels. AITD was defined according to the positivity of TPOAb and TgAb as well as thyroid ultrasonography (US) findings. Results. After full adjustment, TPOAb and/or TgAb positivity (TPO/TgAb (+)) was significantly associated with higher BMI, waist circumference (WC), and HbA1c only in women $(P=0.004,0.026$ and 0.032 , respectively), while both TPO/TgAb positivity and US positivity (TPO/TgAb $(+)$ and US $(+))$ were positively associated with BMI and WC in both genders $(P=0.002$ and 0.020 in men; $P<0.001$ and $<0.001$ in women). TPO/TgAb $(+)$ and US $(+)$ were positively associated with HOMA-IR in women $(P=0.021)$ as well. Binary logistic analysis showed that AITDs had increased risks of central obesity, hyperlipidemia, and metabolic syndrome only in women (all $P<0.05)$. Moreover, TPO/TgAb $(+)$ and US $(+)$ were associated with an increased risk of obesity for both genders $(P=0.014$ in men and $P=0.006$ in women). Conclusions. Thyroid autoimmunity was positively associated with HbA1c, HOMA-IR, obesity, central obesity, hyperlipidemia, and metabolic syndrome, especially in women. This highlighted that AITDs may be potential risk factors for cardiometabolic disorders even if one's TSH was within the reference range.
\end{abstract}

\section{Introduction}

Autoimmune thyroid diseases (AITDs) comprise the most common autoimmune disease in humans. The basic mechanisms in the development of thyroid autoimmunity may be due to a combined TPO- and Tg-specific cytotoxic immune response [1]. High levels of either TPOAb or TgAb serve as a clinical marker for the detection of AITD [1-3] and they can be found in up to $20 \%$ in the "normal" population [4]. AITDs characterized by progressive destruction of thyroid tissue that may raise the level of TSH gradually lead to subclinical hypothyroidism and hypothyroidism $[2,5]$.

Thyroid hormones play important roles in regulating various processes of lipid and glucose metabolism, blood pressure, and energy expenditure [6]. The high prevalence of thyroid dysfunction including overt and subclinical hypothyroidism in the population has considerable consequences for a number of health issues, including insulin resistance, metabolic syndrome, dyslipidemia, central adiposity, and obesity which were involved as contributing factors to cardiometabolic risk 
(CVR) [7-10]. Recently, research has reported that there exists a close relationship of mild thyroid-stimulating hormone (TSH) variations within the reference range with a worse lipid profile, higher BMI, higher blood pressure, and the presence of metabolic syndrome in the general population [11-13]; however, conflicting studies were also present $[14,15]$.

Currently, most of the studies concerning the thyroid tissue and CVR focused on thyroid hormones (i.e., $\mathrm{TSH}, \mathrm{FT}_{3}$, and $\mathrm{FT}_{4}$ ) instead of the direct role of thyroid autoimmunity (i.e., TPOAb positivity and TgAb positivity). The inflammatory pathway has a central role in the pathogenesis of both CVR and AITD. We hypothesize that there may exist common pathways between these two diseases. The association between thyroid autoimmunity and CVR is complex and has not been confirmed. The data used in this study was from a large investigation, the Survey on Prevalence in East China for Metabolic Diseases and Risk Factors (SPECT-China), which was performed in 2014-2015. The purpose of this study was to determine whether the prevalence of AITD was independently associated with CVR in a large Chinese population with normal TSH.

\section{Materials and Methods}

2.1. Study Participants. SPECT-China [16] is a populationbased survey on the prevalence of metabolic diseases and risk factors in East China with a registration number of ChiCTR-ECS-14005052 (http://www.chictr.org.cn). A stratified and cluster-sampling method was used. From February 2014 to December 2015 [17, 18], this study was performed in Shanghai, Zhejiang, Jiangxi, Jiangsu, and Anhui Provinces, for a total of 22 sites in East China. Adults aged 18 years or older who were Chinese citizens and had lived in their current residence for more than 6 months were invited to participate in our study. Those with severe communication problems or severe/acute illness or who were unwilling to participate were excluded. All participants provided written informed consent before data collection. The study protocol was approved by the Ethics Committee of Shanghai Ninth People's Hospital, Shanghai Jiaotong University School of Medicine. All procedures followed were in accordance with the ethical standards of the responsible committee on human experimentation (institutional and national) and with the Helsinki Declaration of 1975, as revised in $2008[17,18]$.

We followed the methods of Chen et al. in 2017 [19]. Our study initially enrolled 10,441 participants above 18 years old $[17,18]$. Participants with missing TSH $(n=45)$, abnormal TSH levels $(\mathrm{TSH}<0.55 \mathrm{mIU} / \mathrm{L}(n=154), \mathrm{TSH}>4.78 \mathrm{mIU} / \mathrm{L}$ $(n=1007))$, had a history that included thyroid surgery or thyroid diseases (including hyperthyroidism, hypothyroidism, subacute thyroiditis, and radioactive iodine treatment history) ( $n=123)$, glucocorticoid treatment $(n=26)$, or missing TPOAb or TgAb levels $(n=4)$ were excluded. Finally, 9082 subjects were included in the final analysis. The inclusion and exclusion of participants in this analysis is shown in Figure 1.
2.2. Data Collection. At every step of this study, all data collection was performed by the same staff from the Department of Endocrinology and Metabolism in Shanghai Ninth People's Hospital, Shanghai Jiaotong University School of Medicine. All staff successfully completed a standard training program that made them familiar with the specific tools and methods used. A standard questionnaire was administered by trained staff to obtain information on demographic characteristics, personal and family medical history, and risk factors in their daily lives. Cardiovascular events including coronary heart disease, myocardial infarct, and stroke were recorded. Weight, height, and waist and hip circumference were measured according to a standard protocol. Blood pressure (BP) was measured at the nondominant arm 3 times consecutively with a 1-minute interval between the measurements with the participant in a seated position after 5 minutes of rest [20]. All anthropometric measurements were conducted at the same time when the serum samples were collected.

2.3. Laboratorial Assays. Serum samples for laboratorial assays were obtained by venipuncture after an 8-hour fast from 0700 to $1000 \mathrm{~h}$ in the morning. Blood samples were stored at $-20^{\circ} \mathrm{C}$ when collected and shipped by air in dry ice to one central laboratory, which was certified by the College of American Pathologists (CAP), within 2-4 hours of collection.

TPOAb, TgAb, TSH, triiodothyronine $\left(\mathrm{T}_{3}\right)$, and thyroxine $\left(\mathrm{T}_{4}\right)$ were measured by the chemiluminescence immunoassay (Siemens, IMMULITE 2000, Erlangen, Germany). Fasting plasma glucose (FPG), low-density lipoprotein (LDL), highdensity lipoprotein (HDL), triglycerides (TG), and total cholesterol (TC) were measured by Beckman Coulter AU680 (Brea, USA). Insulin was detected by chemiluminescence method (Abbott ARCHITECT i2000SR, Chicago, USA). Glycated hemoglobin (HbAlc) was assessed by highperformance liquid chromatography (MQ-2000PT, Medconn, Shanghai, China).

2.4. Thyroid Ultrasonography. Thyroid ultrasound examination was performed by the same registered physicians, who had a professional certificate for ultrasonography awarded by the Ministry of Health of China, using B-mode US imaging (M7 Premium, Shenzhen Mindray Bio-Medical Electronics Co. Ltd, P.R. China).

2.5. Definition of Variables. The normal reference range for TSH is $0.55-4.78 \mathrm{mIU} / \mathrm{L}$; for TPOAb, it is $0-60 \mathrm{IU} / \mathrm{mL}$; and for $\mathrm{TgAb}$, it is $0-60 \mathrm{IU} / \mathrm{mL}$.

AITD was defined as the serum TPOAb and/or TgAb positivity $(>60 \mathrm{IU} / \mathrm{mL})$ (TPO/TgAb $(+))$ and TPOAb and/ or $\mathrm{TgAb}$ positivity together with characteristic ultrasonographic features (diffuse parenchymal hypoechogenicity and/or heterogeneous echogenic pattern of the thyroid gland) (TPO/TgAb (+) and US (+)) $[18,21,22]$ for sensitive analysis.

Central obesity was defined as a waist circumference $\geq 80 \mathrm{~cm}$ in females and $\geq 90 \mathrm{~cm}$ in males [20]. Obesity was defined based upon BMI measures $\geq 30 \mathrm{~kg} / \mathrm{m}^{2}$ [20]. 


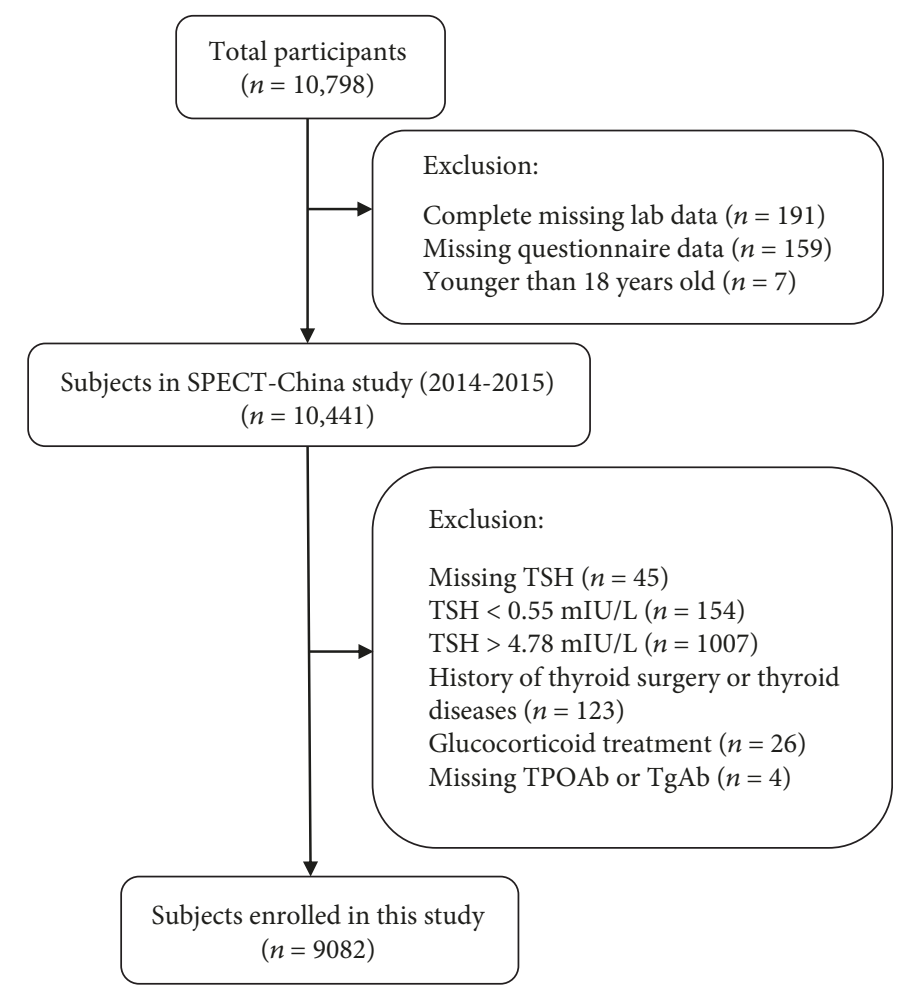

FIgURE 1: Flowchart of participants' inclusion and exclusion.

Based on the American Diabetes Association 2014 criteria, diabetes was defined as a previous diagnosis by healthcare professionals, fasting plasma glucose $\geq 7.0 \mathrm{mmol} / \mathrm{L}$, or $\mathrm{HbAlc} \geq 6.5 \%$. Hyperlipidemia was defined as total cholesterol $\geq 6.22 \mathrm{mmol} / \mathrm{L}$, triglycerides $\geq 2.26 \mathrm{mmol} / \mathrm{L}, \mathrm{LDL}-\mathrm{C} \geq 4.14 \mathrm{mmol} / \mathrm{L}$ or HDL$\mathrm{C}<1.04 \mathrm{mmol} / \mathrm{L}$, or a self-reported previous diagnosis of hyperlipidemia by physicians. Hypertension was defined as a systolic blood pressure of $140 \mathrm{mmHg}$ or higher or a diastolic blood pressure of $90 \mathrm{mmHg}$ or higher or current use of antihypertensive treatment. Metabolic syndrome (MS) was defined based on the International Diabetes Federation criteria (2005). A person with MS must have abdominal obesity (waist circumference: male $\geq 90 \mathrm{~cm}$, female $\geq 80 \mathrm{~cm}$, or $\mathrm{BMI} \geq 30 \mathrm{~kg} / \mathrm{m}^{2}$ ) plus any two of the following four parameters: (1) raised TG $\geq 1.7 \mathrm{mmol} / \mathrm{L}$, or treatment for this dyslipidemia; (2) reduced $\mathrm{HDL}<1.03 \mathrm{mmol} / \mathrm{L}$ in men or $\mathrm{HDL}<1.29 \mathrm{mmol} /$ $\mathrm{L}$ in women, or treatment for this dyslipidemia; (3) raised blood pressure: systolic blood pressure $\geq 130$ or diastolic blood pressure $\geq 85 \mathrm{mmHg}$, or treatment of hypertension; and (4) raised fasting plasma glucose $\geq 5.6 \mathrm{mmol} / \mathrm{L}$ or a history of type 2 diabetes [23].

2.6. Statistical Analysis. We performed survey analyses with IBM SPSS Statistics, Version 22 (IBM Corporation, Armonk, NY, USA). All analyses were two sided. A $P$ value $<0.05$ was considered significant. Continuous variables were expressed as the mean ( \pm standard deviation) values, and categorical variables were presented as numbers (percentage). Continuous variables were compared using Student's $t$-test. The Mann-Whitney $U$ test was used for nonnormally distributed continuous variables, and the Pearson $\chi^{2}$ test was used for dichotomous variables. The body mass index (BMI) was calculated as weight in kilograms divided by height in meters squared. Insulin resistance was estimated by the homeostatic model assessment (HOMA-IR) index: [fasting insulin $(\mathrm{mIU} / \mathrm{L})] \times[\mathrm{FPG}(\mathrm{mmol} / \mathrm{L})] / 22.5$.

Associations among AITD and cardiometabolic risk factors were analyzed using linear regression models with each measure as the outcome. The regression models were adjusted for age, smoking history (including current and past), TSH, BMI (but not including obesity, central obesity, or metabolic syndrome in the regression model), and menopause status (age 50 used as a cutoff for menopause). TG, HbAlc, FPG, and HOMA-IR were ln transformed because of their skewed distribution. The results were expressed as $B$ value and $95 \%$ confidence intervals (CIs).

The associations among AITD and cardiometabolic diseases (categorical variables) were assessed by logistic regression. The regression models were adjusted for age, smoking history (including current and past), TSH, BMI (but not included for obesity, central obesity or metabolic syndrome in the regression model), and menopause status (age 50 used as a cutoff for menopause). Results were expressed as odds ratios (95\% confidence interval).

Sensitivity analyses were performed for the TPO/TgAb $(+)$ and US (+) group.

\section{Results}

3.1. Clinical Characteristics according to TPOAb and TgAb Levels. A total of 9082 subjects (3948 males; 5134 females) with normal TSH levels were enrolled in this study. The 
mean age was $53.19 \pm 13.13$ years, and the mean body mass index (BMI) was $24.48 \pm 3.51 \mathrm{~kg} / \mathrm{m}^{2}$. The prevalence of $\mathrm{TPOAb}$ and/or TgAb positive (TPO/TgAb (+)) was $16.0 \%$ ( $10.1 \%$ in men and $20.6 \%$ in women). The characteristics of the study subjects in terms of TPO/TgAb positivity are summarized in Table 1. For men, participants with AITD had a significantly higher level of TSH and $\mathrm{T}_{4}$, a higher prevalence of subjects with cardiovascular events, and lower percentages of subjects with smoking history compared with participants in the TPOAb and TgAb negative (TPO and TgAb (-)) group. For women, participants with AITD had significantly higher BMI and waist circumference (WC), higher levels of TSH, T 4 , HOMA-IR, HbA1c, and triglycerides (TG), lower percentages of subjects with smoking history, and a higher prevalence of hyperlipidemia and metabolic syndrome.

\subsection{Association of TPO/TgAb Positivity with Cardiometabolic}

Risk Factors. Table 2 summarizes the results of the linear regression models studying the association of TPO/TgAb positivity with cardiometabolic risk factors. TG, HbA1c, FPG, and HOMA-IR were all In transformed because of their skewed distribution. After full adjustment for age, smoking history (including current and past), TSH, BMI (not included for BMI and waist circumference in regression model), and menopause status, TPO/TgAb positivity was significantly associated with BMI (B 0.353, 95\% CI 0.112, 0.595), WC ( $B$ 0.712, 95\% CI 0.086, 1.339), and HbA1c (B 0.010, 95\% CI $0.001,0.019)$ only in female participants, although no significant differences in this regard were noted in male subjects. Moreover, there was no significant association of TPO/TgAb positivity with HDL, LDL, TC, TG, FPG, HOMA-IR, and systolic blood pressure in both genders $(P>0.05)$.

\subsection{Association of TPO/TgAb Positivity with Cardiometabolic} Diseases. Given that the findings of $\mathrm{TPO} / \mathrm{TgAb}$ positivity were positively associated with cardiometabolic risk factors, we evaluated the adjusted odds ratios (ORs) for cardiometabolic disease in the TPO/TgAb (+) group. Adjusted ORs were calculated after adjusting for age, smoking history (including current and past), TSH, BMI (but not included for obesity, central obesity, or metabolic syndrome in the regression model), and menopause status using the binary logistic regression model. As shown in Figure 2(a), TPO/TgAb positivity was associated with an increased risk of central obesity, hyperlipidemia, and metabolic syndrome in women. The ORs were 1.222 (95\% CI 1.050, 1.423, $P=0.010), 1.204$ (95\% CI 1.027, 1.412, $P=0.022$ ), and 1.256 (95\% CI 1.064 , $1.483, P=0.007)$, respectively. Obesity, diabetes, hypertension, and cardiovascular events had no significant relationship with $\mathrm{TPO} / \mathrm{TgAb}$ positivity. No significant association of TPO/TgAb positivity with cardiometabolic diseases were found in male subjects.

3.4. Sensitivity Analyses. After excluding 269 participants missing thyroid US information, 8813 participants (3811 males and 5002 females) were assessed for sensitivity analyses. The prevalence of TPOAb and/or TgAb positive together with characteristic US features (TPO/TgAb (+) and US (+)) was $7.4 \%$ (3.5\% in men and $10.34 \%$ in women). The characteristics of the study subjects in terms of TPO/TgAb positivity and US positivity are summarized in Table 3.

$\mathrm{TPO} / \mathrm{TgAb}$ positivity and US positivity were significantly associated with BMI in both genders (male: $B$ 0.918, 95\% CI $0.331,1.504$; female: $B 0.77995 \%$ CI $0.456,1.102)$ and $\mathrm{WC}$ (male: $B$ 1.998, 95\% CI 0.321, 3.674; female: B $2.10095 \% \mathrm{CI}$ $1.263,2.936)$. For women, TPO/TgAb positivity and US positivity were also significantly associated with HOMA-IR ( $B$ $0.071,95 \%$ CI $0.010,0.132$ ). No other cardiometabolic risk factors were significantly associated with TPO/TgAb positivity and US positivity. (Table 2).

In terms of the relationship between $\mathrm{TPO} / \mathrm{TgAb}(+)$ and US (+) and cardiometabolic diseases, the result was similar to the TPO/TgAb (+) group. Women with TPO/TgAb positivity and US positivity had a greater risk of central obesity, hyperlipidemia, and metabolic syndrome. The ORs were 1.554 (95\% CI 1.269, 1.902, $P<0.001), 1.260$ (95\% CI $1.024,1.551, P=0.029$ ), and 1.520 (95\% CI 1.230, 1.879, $P<0.001$ ), respectively (Figure $2(\mathrm{~b})$ ). Moreover, TPO/TgAb positivity and US positivity also had a significantly association with obesity in both genders (male: OR 1.982, 95\% CI $1.149,3.420, P=0.014$; female: OR 1.601, 95\% CI 1.145, 2.238, $P=0.006)$.

\section{Discussion}

Our present evaluation of the association of thyroid autoimmunity with cardiometabolic diseases and risk factors according to the TPOAb and (or) TgAb positivity and US findings has revealed that thyroid autoimmunity played an independent role in cardiometabolic disorders, especially in women.

The Tehran Lipid and Glucose Study [24] showed that the frequency of positive TPOAb in never smokers was significantly higher than ever smokers. Similarly, our data showed that the frequency of smoke was decreased in participants with AITD. A significant difference was detected in women. Smoking has significant effects on thyroid function and could be a potential confounder to assess the association between AITD and metabolic syndrome [24, 25]. In this study, all analyses were performed with adjustment for smoking.

Only scanty studies about the association of thyroid autoimmunity with obesity and central obesity in a large Chinese population with a normal TSH level were available. This study showed that thyroid autoimmunity was positively associated with BMI and WC in women. Obesity and central obesity were increased by about 1.60 - and 1.55 -fold in the presence of both TPO/TgAb positivity and US positivity. For men, the adjusted odds ratio of obesity increased by $98 \%$ in the TPO/TgAb (+) and US (+) group. Thus, our data suggested that thyroid autoimmunity had a close relationship with obesity and central obesity.

We also found in women that both TPO/TgAb (+) patients and TPO/TgAb $(+)$ together with US $(+)$ patients had higher serum TC, TG, and LDL levels and lower HDL levels, but only TG had a statistically significant change. Besides these lipid profiles, previous diagnosis of hyperlipidemia was also considered in this study. After full adjustment, 
TABLE 1: Characteristics of subjects in terms of the level of serum TPOAb and TgAb.

\begin{tabular}{|c|c|c|c|c|}
\hline & Total & $\mathrm{TPO}$ and $\mathrm{TgAb}(-)$ & $\mathrm{TPO} / \mathrm{TgAb}(+)$ & $P$ value \\
\hline \multicolumn{5}{|l|}{ Men } \\
\hline$N(\%)$ & $3948(100)$ & $3548(89.9)$ & $400(10.1)$ & - \\
\hline Age (year) & $53.93 \pm 13.12$ & $53.91 \pm 13.05$ & $54.13 \pm 13.82$ & 0.753 \\
\hline Smokers (\%) & 55.3 & 55.8 & 51.3 & 0.095 \\
\hline Systolic BP (mmHg) & $134.31 \pm 20.66$ & $134.41 \pm 20.80$ & $133.42 \pm 19.45$ & 0.347 \\
\hline BMI $\left(\mathrm{kg} / \mathrm{m}^{2}\right)$ & $24.82 \pm 3.35$ & $24.80 \pm 3.35$ & $25.04 \pm 3.36$ & 0.174 \\
\hline $\mathrm{WC}(\mathrm{cm})$ & $84.23 \pm 9.60$ & $84.22 \pm 9.60$ & $84.37 \pm 9.55$ & 0.777 \\
\hline FPG (mmol/L) & $5.70 \pm 1.52$ & $5.72 \pm 1.56$ & $5.55 \pm 1.15$ & 0.481 \\
\hline HOMA-IR & $1.47 \pm 2.21$ & $1.48 \pm 2.30$ & $1.32 \pm 1.15$ & 0.461 \\
\hline HbAlc (\%) & $5.61 \pm 1.01$ & $5.62 \pm 1.02$ & $5.52 \pm 0.91$ & 0.112 \\
\hline LDL (mmol/L) & $3.07 \pm 0.76$ & $3.07 \pm 0.77$ & $3.06 \pm 0.69$ & 0.807 \\
\hline $\mathrm{HDL}(\mathrm{mmol} / \mathrm{L})$ & $1.36 \pm 0.32$ & $1.36 \pm 0.32$ & $1.35 \pm 0.30$ & 0.557 \\
\hline $\mathrm{TG}(\mathrm{mmol} / \mathrm{L})$ & $1.88 \pm 1.95$ & $1.90 \pm 2.02$ & $1.74 \pm 1.19$ & 0.856 \\
\hline $\mathrm{TC}(\mathrm{mmol} / \mathrm{L})$ & $5.15 \pm 1.13$ & $5.16 \pm 1.14$ & $5.11 \pm 1.00$ & 0.400 \\
\hline TSH (mIU/L) & $2.13 \pm 0.92$ & $2.11 \pm 0.91$ & $2.36 \pm 0.99$ & $<0.001$ \\
\hline $\mathrm{T}_{3}(\mathrm{nmol} / \mathrm{L})$ & $1.79 \pm 0.53$ & $1.77 \pm 0.42$ & $1.94 \pm 1.10$ & 0.152 \\
\hline $\mathrm{T}_{4}(\mathrm{nmol} / \mathrm{L})$ & $113.87 \pm 22.34$ & $113.64 \pm 22.42$ & $116.00 \pm 21.43$ & 0.047 \\
\hline Hyperlipidemia (\%) & 42.2 & 42.2 & 42.3 & 0.972 \\
\hline Diabetes (\%) & 14.9 & 15.1 & 13.5 & 0.392 \\
\hline Hypertension (\%) & 51.3 & 51.7 & 48.2 & 0.193 \\
\hline Metabolic syndrome (\%) & 20.3 & 20.4 & 19.3 & 0.604 \\
\hline Cardiovascular events (\%) & 7.4 & 7.1 & 10.3 & 0.021 \\
\hline \multicolumn{5}{|l|}{ Women } \\
\hline$N(\%)$ & $5134(100)$ & $4077(79.4)$ & $1057(20.6)$ & - \\
\hline Age (year) & $52.62 \pm 13.11$ & $52.56 \pm 13.20$ & $52.83 \pm 12.77$ & 0.562 \\
\hline Smokers (\%) & 2.8 & 3.0 & 1.8 & 0.036 \\
\hline Systolic BP (mmHg) & $130.19 \pm 22.28$ & $129.99 \pm 22.06$ & $130.97 \pm 23.11$ & 0.207 \\
\hline BMI $\left(\mathrm{kg} / \mathrm{m}^{2}\right)$ & $24.21 \pm 3.60$ & $24.13 \pm 3.56$ & $24.54 \pm 3.74$ & 0.002 \\
\hline $\mathrm{WC}(\mathrm{cm})$ & $77.71 \pm 9.99$ & $77.54 \pm 10.00$ & $78.38 \pm 9.92$ & 0.017 \\
\hline $\mathrm{FPG}(\mathrm{mmol} / \mathrm{L})$ & $5.56 \pm 1.34$ & $5.56 \pm 1.32$ & $5.57 \pm 1.42$ & 0.623 \\
\hline HOMA-IR & $1.56 \pm 1.95$ & $1.53 \pm 1.77$ & $1.67 \pm 2.54$ & 0.017 \\
\hline HbAlc (\%) & $5.45 \pm 0.90$ & $5.43 \pm 0.88$ & $5.52 \pm 0.96$ & 0.001 \\
\hline $\mathrm{LDL}(\mathrm{mmol} / \mathrm{L})$ & $3.06 \pm 0.79$ & $3.05 \pm 0.79$ & $3.10 \pm 0.81$ & 0.096 \\
\hline $\mathrm{HDL}(\mathrm{mmol} / \mathrm{L})$ & $1.48 \pm 0.31$ & $1.48 \pm 0.32$ & $1.47 \pm 0.31$ & 0.313 \\
\hline $\mathrm{TG}(\mathrm{mmol} / \mathrm{L})$ & $1.48 \pm 1.14$ & $1.46 \pm 1.04$ & $1.58 \pm 1.47$ & 0.003 \\
\hline $\mathrm{TC}(\mathrm{mmol} / \mathrm{L})$ & $5.14 \pm 1.13$ & $5.15 \pm 1.16$ & $5.15 \pm 1.03$ & 0.994 \\
\hline TSH (mIU/L) & $2.46 \pm 1.00$ & $2.42 \pm 1.00$ & $2.59 \pm 1.02$ & $<0.001$ \\
\hline $\mathrm{T}_{3}(\mathrm{nmol} / \mathrm{L})$ & $1.73 \pm 0.40$ & $1.72 \pm 0.36$ & $1.77 \pm 0.50$ & 0.018 \\
\hline $\mathrm{T}_{4}(\mathrm{nmol} / \mathrm{L})$ & $115.49 \pm 19.59$ & $115.17 \pm 19.20$ & $116.73 \pm 20.99$ & 0.029 \\
\hline Hyperlipidemia (\%) & 31.3 & 30.4 & 34.7 & 0.006 \\
\hline Diabetes (\%) & 12.0 & 11.7 & 13.1 & 0.230 \\
\hline Hypertension (\%) & 41.1 & 40.8 & 42.1 & 0.449 \\
\hline Metabolic syndrome (\%) & 26.5 & 25.6 & 29.8 & 0.007 \\
\hline Cardiovascular events (\%) & 7.5 & 7.3 & 8.5 & 0.168 \\
\hline
\end{tabular}

$P$ value: $\mathrm{TPO} / \mathrm{TgAb}(+)$ versus TPO and TgAb (-). Data are presented as the mean \pm standard deviation for continuous variables or as a percentage (\%) for categorical variables. BP, blood pressure; BMI, body mass index; WC, waist circumference; FPG, fasting blood glucose; HOMA-IR, homeostasis model assessment of insulin resistance; HbAlc, glycated hemoglobin; LDL, low-density lipoprotein; HDL, high-density lipoprotein; TG, triglycerides; TC, total cholesterol; $\mathrm{TSH}$, thyroid-stimulating hormone; $\mathrm{T}_{3}$, triiodothyronine; $\mathrm{T}_{4}$, thyroxin. 
TABLE 2: Associations of AITD with cardiometabolic risk factors by linear regression.

\begin{tabular}{lcccccccc}
\hline & & \multicolumn{3}{c}{ Men } & \multicolumn{3}{c}{ Women } \\
& $B(95 \% \mathrm{CI})$ & $P$ & $B(95 \% \mathrm{CI})$ & $P$ & $B(95 \%$ CI $)$ & $P$ & $B(95 \%$ CI $)$ \\
\hline BMI & $0.171(-0.186,0.527)$ & 0.349 & $0.918(0.331,1.504)$ & 0.002 & $0.353(0.112,0.595)$ & 0.004 & $0.779(0.456,1.102)$ & $<0.001$ \\
WC & $0.097(-0.925,1.120)$ & 0.852 & $1.998(0.321,3.674)$ & 0.020 & $0.712(0.086,1.339)$ & 0.026 & $2.100(1.263,2.936)$ & $<0.001$ \\
HDL & $-0.004(-0.037,0.028)$ & 0.792 & $0.011(-0.042,0.064)$ & 0.691 & $0.001(-0.021,0.022)$ & 0.950 & $-0.004(-0.033,0.024)$ & 0.778 \\
LDL & $-0.004(-0.046,0.006)$ & 0.924 & $0.021(-0.107,0.149)$ & 0.749 & $0.026(-0.026,0.078)$ & 0.331 & $-0.011(-0.081,0.059)$ & 0.751 \\
TC & $-0.030(-0.150,0.090)$ & 0.625 & $0.066(-0.130,0.262)$ & 0.508 & $-0.017(-0.092,0.059)$ & 0.662 & $-0.020(-0.121,0.081)$ & 0.700 \\
TG & $-0.033(-0.092,0.027)$ & 0.281 & $-0.021(-0.119,0.076)$ & 0.667 & $0.032(0.000,0.065)$ & 0.052 & $0.035(-0.009,0.078)$ & 0.121 \\
HbA1c & $-0.014(-0.030,0.002)$ & 0.093 & $-0.004(-0.030,0.023)$ & 0.785 & $0.010(0.001,0.019)$ & 0.032 & $0.011(-0.002,0.023)$ & 0.089 \\
FPG & $-0.021(-0.042,0.000)$ & 0.055 & $0.006(-0.028,0.041)$ & 0.724 & $-0.005(-0.017,0.007)$ & 0.444 & $0.003(-0.013,0.019)$ & 0.718 \\
HOMA-IR & $-0.011(-0.083,0.062)$ & 0.771 & $0.058(-0.061,0.177)$ & 0.340 & $0.032(-0.013,0.077)$ & 0.159 & $0.071(0.010,0.132)$ & 0.021 \\
Systolic BP & $-1.509(-3.536,0.519)$ & 0.145 & $-1.071(-4.418,2.276)$ & 0.531 & $0.173(-1.159,1.505)$ & 0.799 & $-0.309(-2.091,1.474)$ & 0.731 \\
\hline
\end{tabular}

$\mathrm{TG}, \mathrm{HbA}_{1} \mathrm{c}, \mathrm{FPG}$, and HOMA-IR was $\ln$ transformed for normal distribution. The regression models were adjusted for age, smoking history (including current and past), TSH, BMI (not included for BMI and WC in regression model), and menopause status in women.

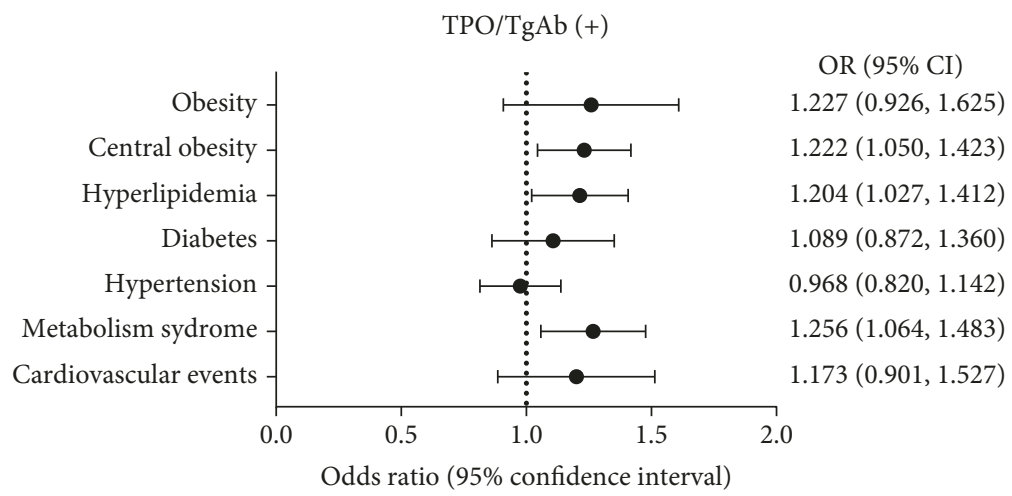

(a)

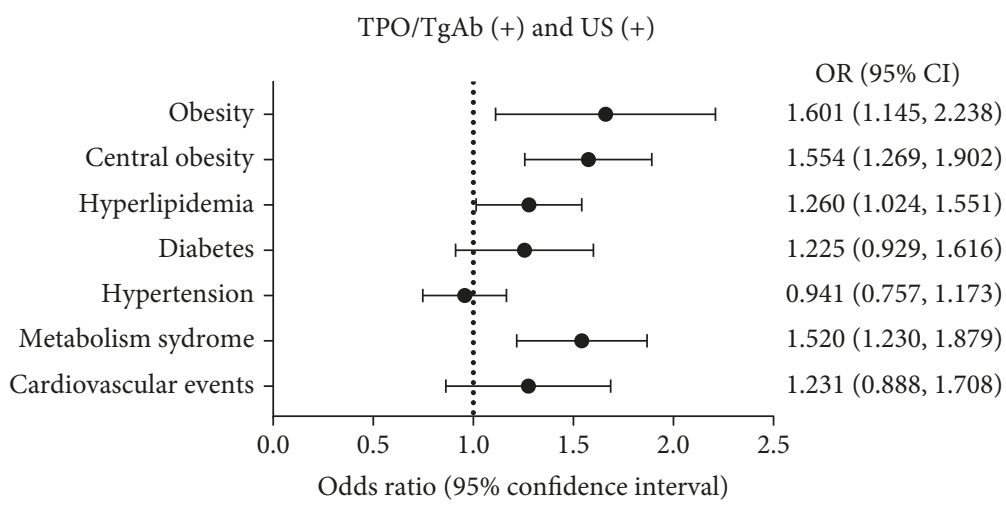

(b)

FIGURE 2: Associations of AITD with cardiometabolic diseases in women. They were analyzed using logistic regression. The regression models were adjusted for age, smoking history (including current and past), TSH, BMI (but not included for obesity or central obesity in the regression model), and menopause status. (a) TPO/TgAb (+) group; (b) TPO/TgAb (+) and US (+) group.

patients with AITD had more risk for hyperlipidemia. Some other studies reached a similar conclusion that positive TPOAb can cause dyslipidemia in the euthyroid population $[26,27]$, while Wells and Hueston recorded no significant difference between the TPOAb-positive group and TPOAbnegative group [28].
In terms of glucose metabolism, several studies have suggested a major role for autoimmunity in the pathogenesis of type 1 diabetes mellitus $\left(\mathrm{T}_{1} \mathrm{DM}\right)$. Thyroid antibodies were frequent in patients with $\mathrm{T}_{1} \mathrm{DM}[29,30]$. A case-control study [31] involving three hundred and two type 2 diabetes mellitus $\left(\mathrm{T}_{2} \mathrm{DM}\right)$ patients and three hundred and ten 
TABLE 3: Characteristic of subjects in terms of TPOAb, TgAb, and US.

\begin{tabular}{|c|c|c|c|}
\hline & TPO and $\operatorname{TgAb}(-)$ & $\mathrm{TPO} / \mathrm{TgAb}(+)$ and US (+) & $P$ value \\
\hline \multicolumn{4}{|l|}{ Men } \\
\hline$N(\%)$ & $3426(89.9)$ & $134(3.5)$ & - \\
\hline Age (year) & $53.80 \pm 13.04$ & $54.52 \pm 13.09$ & 0.527 \\
\hline Smokers (\%) & 55.6 & 49.6 & 0.177 \\
\hline Systolic BP (mmHg) & $134.36 \pm 20.81$ & $135.56 \pm 20.68$ & 0.516 \\
\hline BMI $\left(\mathrm{kg} / \mathrm{m}^{2}\right)$ & $24.83 \pm 3.35$ & $25.75 \pm 3.45$ & 0.002 \\
\hline $\mathrm{WC}(\mathrm{cm})$ & $84.26 \pm 9.60$ & $86.21 \pm 9.29$ & 0.022 \\
\hline $\mathrm{FPG}(\mathrm{mmol} / \mathrm{L})$ & $5.72 \pm 1.56$ & $5.22 \pm 1.18$ & 0.185 \\
\hline HOMA-IR & $1.49 \pm 2.32$ & $1.43 \pm 0.98$ & 0.038 \\
\hline HbAlc (\%) & $5.62 \pm 1.02$ & $5.61 \pm 0.89$ & 0.721 \\
\hline $\mathrm{LDL}(\mathrm{mmol} / \mathrm{L})$ & $3.07 \pm 0.76$ & $3.14 \pm 0.68$ & 0.351 \\
\hline $\mathrm{HDL}(\mathrm{mmol} / \mathrm{L})$ & $1.36 \pm 0.32$ & $1.34 \pm 0.31$ & 0.543 \\
\hline $\mathrm{TG}(\mathrm{mmol} / \mathrm{L})$ & $1.91 \pm 1.99$ & $1.84 \pm 1.22$ & 0.187 \\
\hline $\mathrm{TC}(\mathrm{mmol} / \mathrm{L})$ & $5.16 \pm 1.13$ & $5.22 \pm 1.18$ & 0.536 \\
\hline TSH (mIU/L) & $2.11 \pm 0.91$ & $2.56 \pm 1.02$ & $<0.001$ \\
\hline $\mathrm{T}_{3}(\mathrm{nmol} / \mathrm{L})$ & $1.77 \pm 0.42$ & $1.73 \pm 0.36$ & 0.127 \\
\hline $\mathrm{T}_{4}(\mathrm{nmol} / \mathrm{L})$ & $113.59 \pm 22.30$ & $110.13 \pm 19.24$ & 0.077 \\
\hline Hyperlipidemia (\%) & 42.7 & 49.3 & 0.130 \\
\hline Diabetes (\%) & 15.0 & 17.9 & 0.363 \\
\hline Hypertension (\%) & 51.6 & 50.8 & 0.852 \\
\hline Metabolic syndrome (\%) & 20.5 & 24.6 & 0.257 \\
\hline Cardiovascular events (\%) & 7.2 & 9.0 & 0.429 \\
\hline \multicolumn{4}{|l|}{ Women } \\
\hline$N(\%)$ & $3970(79.4)$ & $517(10.3)$ & - \\
\hline Age (year) & $52.45 \pm 13.16$ & $54.56 \pm 12.31$ & $<0.001$ \\
\hline Smokers (\%) & 2.9 & 1.4 & 0.053 \\
\hline Systolic BP (mmHg) & $129.93 \pm 22.07$ & $132.72 \pm 23.65$ & 0.008 \\
\hline BMI $\left(\mathrm{kg} / \mathrm{m}^{2}\right)$ & $24.16 \pm 3.56$ & $25.11 \pm 3.73$ & 0.002 \\
\hline $\mathrm{WC}(\mathrm{cm})$ & $77.53 \pm 10.00$ & $80.32 \pm 10.05$ & $<0.001$ \\
\hline $\mathrm{FPG}(\mathrm{mmol} / \mathrm{L})$ & $5.55 \pm 1.30$ & $5.67 \pm 1.53$ & 0.061 \\
\hline HOMA-IR & $1.53 \pm 1.77$ & $1.78 \pm 2.86$ & $<0.001$ \\
\hline HbA1c (\%) & $5.43 \pm 0.87$ & $5.59 \pm 0.99$ & $<0.001$ \\
\hline $\mathrm{LDL}(\mathrm{mmol} / \mathrm{L})$ & $3.05 \pm 0.79$ & $3.11 \pm 0.81$ & 0.084 \\
\hline $\mathrm{HDL}(\mathrm{mmol} / \mathrm{L})$ & $1.48 \pm 0.32$ & $1.46 \pm 0.31$ & 0.088 \\
\hline $\mathrm{TG}(\mathrm{mmol} / \mathrm{L})$ & $1.46 \pm 1.04$ & $1.71 \pm 1.88$ & $<0.001$ \\
\hline $\mathrm{TC}(\mathrm{mmol} / \mathrm{L})$ & $5.14 \pm 1.16$ & $5.20 \pm 1.03$ & 0.280 \\
\hline TSH (mIU/L) & $2.43 \pm 0.99$ & $2.68 \pm 1.05$ & $<0.001$ \\
\hline $\mathrm{T}_{3}(\mathrm{nmol} / \mathrm{L})$ & $1.72 \pm 0.36$ & $1.75 \pm 0.53$ & 0.644 \\
\hline $\mathrm{T}_{4}(\mathrm{nmol} / \mathrm{L})$ & $115.10 \pm 19.16$ & $115.31 \pm 21.45$ & 0.833 \\
\hline Hyperlipidemia (\%) & 30.4 & 38.9 & $<0.001$ \\
\hline Diabetes (\%) & 11.5 & 15.7 & 0.006 \\
\hline Hypertension (\%) & 40.6 & 46.4 & 0.013 \\
\hline Metabolic syndrome (\%) & 25.5 & 35.8 & $<0.001$ \\
\hline Cardiovascular events (\%) & 7.4 & 10.1 & 0.033 \\
\hline
\end{tabular}

Data are presented as the mean \pm standard deviation for continuous variables or as a percentage (\%) for categorical variables. BP, blood pressure; BMI, body mass index; WC, waist circumference; FPG, fasting blood glucose; HOMA-IR, homeostasis model assessment of insulin resistance; HbA1c, glycated hemoglobin; LDL, low-density lipoprotein; HDL, high-density lipoprotein; TG, triglycerides; TC, total cholesterol; TSH, thyroid-stimulating hormone; $\mathrm{T}_{3}$, triiodothyronine; $\mathrm{T}_{4}$, thyroxin. 
nondiabetic controls showed that TPOAb level was significantly higher among $\mathrm{T}_{2} \mathrm{DM}$ subjects compared to controls, and thyroid autoimmunity in $\mathrm{T}_{2} \mathrm{DM}$ subjects was significantly associated with poor glycemic control. In line with this study, our data showed in women that the TPO/TgAb (+) was positively associated with $\mathrm{HbAlc}$ and TPO/TgAb and US (+) was positively associated with HOMA-IR.

Metabolic syndrome is an important predictor of cardiometabolic risk and is a cluster of abnormalities such as obesity (including central obesity), dyslipidemia, high plasma glucose, and hypertension [32]. The findings of female gender dominance in the prevalence of metabolic syndrome were seen in several studies $[33,34]$ which showed the similar tendency with the prevalence of AITD [35]. In addition, higher TSH and IL- 6 concentrations were detected in metabolic syndrome [36] while AITD was reported to be associated with higher TSH and IL-6 levels as well $[1,5]$. We therefore hypothesized that there may be a crosstalk between AITD and metabolic syndrome. Our present findings suggested that women with AITD had a greater risk of metabolic syndrome. The adjusted odds ratio of metabolic syndrome was increased by about $25.6 \%$ and $52.0 \%$ in the TPO/TgAb (+) group and $\mathrm{TPO} / \mathrm{TgAb}(+)$ and US (+) group. However, conflicting evidence has been reported. A study including three hundred and seventy-two postmenopausal women showed that the prevalence of metabolic syndrome was similar between the TPOAb-positive group and the TPOAbnegative group in euthyroid women. Obese subclinical hypothyroid women with Hashimoto's thyroditis have a higher prevalence of metabolic syndrome [36]. Agbaht et al. pointed out from their study including five hundred and eighty-four participants that TPOAb level had no association with metabolic syndrome, even in the obese population [37]. In terms of different ages, race, and ethnicity, gender composition may have accounted for the difference in results obtained. Additionally, compared with the above studies, our survey with such a large sample size maybe more reliable.

Our results confirmed a close relationship between AITD and cardiometabolic disorders, especially in women. In general, autoimmune diseases including AITD are much more common in females [38], and the immune response is greater in females than in males as well [39]. It is well established that the immune system exhibits sexual dimorphism in humans. That is, AITD affects males and females differently [40, 41].

Our study has several strengths. First, we evaluated a relatively large sample of participants to examine the association of TPOAb and $\mathrm{TgAb}$ with cardiometabolic risks. Second, the data were highly reliable because all the anthropometric measurements and questionnaires were completed by the same trained research group using strong quality control, and all the tests were performed with the same equipment and protocol. Third, compared to a clinic-based population, community-dwelling participants living in multiple sites in China were recruited so that the results may be more representative.

However, several limitations of this study should also be considered. First, because of the cross-sectional study, the direction of any causal relationship could not be established. Prospective studies are needed. Second, we used an age proxy to define postmenopausal status. In China, the overall median age at natural menopause is 50 years [42]. Third, no association between AITDs and cardiovascular events was found in this study. However, it must be noted that those with severe communication problems or severe/acute illness (maybe a result from these cardiovascular events) were excluded in this study, which may have introduced bias. Fourth, we did not measure levels of $\mathrm{FT}_{3}$ and $\mathrm{FT}_{4}$ and thus could not assess their probable role in metabolic syndrome parameters.

\section{Conclusion}

Thyroid autoimmunity was positively associated with $\mathrm{HbAlc}$, HOMA-IR, central obesity, hyperlipidemia, and metabolic syndrome, especially in women. It was also positively associated with obesity in both genders. This highlighted that AITD may be a potential independent risk factor for cardiometabolic disorders even if one's TSH was among the reference range.

\section{Abbreviations}

$\begin{array}{ll}\text { TPOAb: } & \text { Thyroid peroxidase antibody } \\ \text { TgAb: } & \text { Thyroglobulin antibody } \\ \text { US: } & \text { Ultrasonography } \\ \text { AITDs: } & \text { Thyroid autoimmune diseases } \\ \text { CVR: } & \text { Cardiometabolic risk } \\ \text { BP: } & \text { Blood pressure } \\ \text { BMI: } & \text { Body mass index } \\ \text { WC: } & \text { Waist circumference } \\ \text { FPG: } & \text { Fasting blood glucose } \\ \text { HOMA-IR: } & \text { Homeostasis model assessment of insulin } \\ & \text { resistance } \\ \text { HbA1c: } & \text { Glycated hemoglobin } \\ \text { LDL: } & \text { Low-density lipoprotein } \\ \text { HDL: } & \text { High-density lipoprotein } \\ \text { TG: } & \text { Triglycerides } \\ \text { TC: } & \text { Total cholesterol } \\ \text { TSH: } & \text { Thyroid-stimulating hormone } \\ \text { T }: & \text { Triiodothyronine } \\ \text { T: } & \text { Thyroxine. }\end{array}$

\section{Data Availability}

The data used to support the findings of this study are available from the corresponding author upon request.

\section{Conflicts of Interest}

The authors declare that they have no competing interests.

\section{Authors' Contributions}

Yingli Lu designed the study. Yi Chen, Chunfang Zhu, Yingchao Chen, Ningjian Wang, Qin Li, Bing Han, Li Zhao, Chi Chen, and Hualing Zhai participated in the acquisition of data. Yingli Lu evaluated the literature. Yi Chen undertook the statistical analysis, wrote the first draft of the manuscript, and revised the manuscript. Yingli Lu and Lijuan Zhang 
edited and revised the manuscript. All authors read and approved the final manuscript for publication.

\section{Acknowledgments}

This study was supported by the National Natural Science Foundation of China (81570726), the Shanghai Jiao Tong University School of Medicine (2014), the Science and Technology Commission of Shanghai Municipality (16411971200), the Commission of Health and Family Planning of Pudong District (PW2015D-5), the Fourth Round of Three-Year Public Health Action Plan of Shanghai by the Foundation of Shanghai Municipal Commission of Health and Family Planning (15GWZK0202), and the Clinical Research Plan of SHDC (16CR3076B).

\section{References}

[1] G. Cogni and L. Chiovato, "An overview of the pathogenesis of thyroid autoimmunity," Hormones, vol. 12, no. 1, pp. 19-29, 2013.

[2] L. Brcic, A. Baric, S. Gracan et al., "Association of established thyroid peroxidase autoantibody (TPOAb) genetic variants with Hashimoto's thyroiditis," Autoimmunity, vol. 49, no. 7, pp. 480-485, 2016.

[3] G. Effraimidis, K. Badenhoop, J. G. P. Tijssen, and W. M. Wiersinga, "Vitamin D deficiency is not associated with early stages of thyroid autoimmunity," European Journal of Endocrinology, vol. 167, no. 1, pp. 43-48, 2012.

[4] M. S. Massoudi, E. N. Meilahn, T. J. Orchard et al., "Prevalence of thyroid antibodies among healthy middle-aged women. Findings from the thyroid study in healthy women," Annals of Epidemiology, vol. 5, no. 3, pp. 229-233, 1995.

[5] S. J. Merrill and Y. Mu, "Thyroid autoimmunity as a window to autoimmunity: an explanation for sex differences in the prevalence of thyroid autoimmunity," Journal of Theoretical Biology, vol. 375, pp. 95-100, 2015.

[6] R. Mullur, Y. Y. Liu, and G. A. Brent, "Thyroid hormone regulation of metabolism," Physiological Reviews, vol. 94, no. 2, pp. 355-382, 2014.

[7] T. Du, G. Yuan, M. Zhang, X. Zhou, X. Sun, and X. Yu, "Clinical usefulness of lipid ratios, visceral adiposity indicators, and the triglycerides and glucose index as risk markers of insulin resistance," Cardiovascular Diabetology, vol. 13, no. 1, p. 146, 2014.

[8] G. R. Hajer, T. W. van Haeften, and F. L. J. Visseren, "Adipose tissue dysfunction in obesity, diabetes, and vascular diseases," European Heart Journal, vol. 29, no. 24, pp. 2959-2971, 2008.

[9] L. H. Duntas and G. Brenta, "The effect of thyroid disorders on lipid levels and metabolism," The Medical Clinics of North America, vol. 96, no. 2, pp. 269-281, 2012.

[10] E. Fommei and G. Iervasi, "The role of thyroid hormone in blood pressure homeostasis: evidence from short-term hypothyroidism in humans," The Journal of Clinical Endocrinology and Metabolism, vol. 87, no. 5, pp. 1996-2000, 2002.

[11] B. O. Åsvold, L. J. Vatten, T. I. L. Nilsen, and T. Bjøro, “The association between TSH within the reference range and serum lipid concentrations in a population-based study. The HUNT study," European Journal of Endocrinology, vol. 156, no. 2, pp. 181-186, 2007.
[12] B. O. Åsvold, T. Bjøro, T. I. L. Nilsen, and L. J. Vatten, "Association between blood pressure and serum thyroidstimulating hormone concentration within the reference range: a population-based study," The Journal of Clinical Endocrinology and Metabolism, vol. 92, no. 3, pp. 841-845, 2007.

[13] S. Ruhla, M. O. Weickert, A. M. Arafat et al., "A high normal TSH is associated with the metabolic syndrome," Clinical Endocrinology, vol. 72, no. 5, pp. 696-701, 2010.

[14] O. Bakiner, E. Bozkirli, G. Cavlak, K. Ozsahin, and E. Ertorer, "Are plasma thyroid-stimulating hormone levels associated with degree of obesity and metabolic syndrome in euthyroid obese patients? A Turkish cohort study," ISRN Endocrinology, vol. 2014, Article ID 803028, 7 pages, 2014.

[15] N. Manji, K. Boelaert, M. C. Sheppard, R. L. Holder, S. C. Gough, and J. A. Franklyn, "Lack of association between serum TSH or free T4 and body mass index in euthyroid subjects," Clinical Endocrinology, vol. 64, no. 2, pp. 125-128, 2006.

[16] N. Wang, J. Cheng, B. Han et al., "Exposure to severe famine in the prenatal or postnatal period and the development of diabetes in adulthood: an observational study," Diabetologia, vol. 60, no. 2, pp. 262-269, 2017.

[17] N. Wang, K. Zhang, B. Han et al., "Follicle stimulating hormone, its novel association with sex hormone binding globulin in men and postmenopausal women," Endocrine, vol. 56, no. 3, pp. 649-657, 2017.

[18] Y. Chen, Y. Chen, F. Xia et al., "A higher ratio of estradiol to testosterone is associated with autoimmune thyroid disease in males," Thyroid, vol. 27, no. 7, pp. 960-966, 2017.

[19] Y. Chen, Y. Chen, N. Wang et al., "Thyroid stimulating hormone within the reference range is associated with visceral adiposity index and lipid accumulation product: a populationbased study of SPECT-China," Hormone and Metabolic Research, vol. 50, no. 1, pp. 29-36, 2018.

[20] Y. Xu, L. Wang, J. He et al., "Prevalence and control of diabetes in Chinese adults," JAMA, vol. 310, no. 9, pp. 948-959, 2013.

[21] Y. M. Choi, W. G. Kim, T. Y. Kim et al., "Low levels of serum vitamin D3 are associated with autoimmune thyroid disease in pre-menopausal women," Thyroid, vol. 24, no. 4, pp. 655-661, 2014.

[22] G. Muscogiuri, D. Mari, S. Prolo et al., "25 hydroxyvitamin $\mathrm{D}$ deficiency and Its relationship to autoimmune thyroid disease in the elderly," International Journal of Environmental Research and Public Health, vol. 13, no. 9, 2016.

[23] K. G. M. M. Alberti, P. Zimmet, and J. Shaw, "The metabolic syndrome-a new worldwide definition," Lancet, vol. 366, no. 9491, pp. 1059-1062, 2005.

[24] L. Mehran, A. Amouzgar, H. Delshad, and F. Azizi, “The association of cigarette smoking with serum TSH concentration and thyroperoxidase antibody," Experimental and Clinical Endocrinology \& Diabetes, vol. 120, no. 2, pp. 80-83, 2012.

[25] R. M. Belin, B. C. Astor, N. R. Powe, and P. W. Ladenson, "Smoke exposure is associated with a lower prevalence of serum thyroid autoantibodies and thyrotropin concentration elevation and a higher prevalence of mild thyrotropin concentration suppression in the third National Health and Nutrition Examination Survey (NHANES III)," The Journal of Clinical Endocrinology and Metabolism, vol. 89, no. 12, pp. 60776086, 2004.

[26] D. Kang, Q. Yin, X. Yan et al., "Serum cholesterol levels in middle-aged euthyroid subjects with positive thyroid 
peroxidase antibodies," International Journal of Clinical and Experimental Medicine, vol. 8, no. 11, pp. 21623-21628, 2015.

[27] O. Topaloglu, F. Gokay, K. Kucukler et al., "Is autoimmune thyroiditis a risk factor for early atherosclerosis in premenopausal women even if in euthyroid status?," Endocrine, vol. 44, no. 1, pp. 145-151, 2013.

[28] B. J. Wells and W. J. Hueston, "Are thyroid peroxidase antibodies associated with cardiovascular disease risk in patients with subclinical hypothyroidism?," Clinical Endocrinology, vol. 62, no. 5, pp. 580-584, 2005.

[29] W. Sakly, A. Mankai, A. Achour et al., "Thyroid-related autoantibodies in Tunisian patients with type 1 diabetes," Endocrine Research, vol. 37, no. 2, pp. 59-66, 2011.

[30] B. Jonsdottir, C. Larsson, A. Carlsson et al., "Thyroid and islet autoantibodies predict autoimmune thyroid disease at type 1 diabetes diagnosis," The Journal of Clinical Endocrinology and Metabolism, vol. 102, no. 4, pp. 1277-1285, 2016.

[31] O. Sarfo-Kantanka, F. S. Sarfo, E. O. Ansah et al., "Frequency and determinants of thyroid autoimmunity in Ghanaian type 2 diabetes patients: a case-control study," BMC Endocrine Disorders, vol. 17, no. 1, p. 2, 2017.

[32] M. A. Michalaki, A. G. Vagenakis, A. S. Leonardou et al., "Thyroid function in humans with morbid obesity," Thyroid, vol. 16, no. 1, pp. 73-78, 2006.

[33] Y. He, B. Jiang, J. Wang et al., "Prevalence of the metabolic syndrome and its relation to cardiovascular disease in an elderly Chinese population," Journal of the American College of Cardiology, vol. 47, no. 8, pp. 1588-1594, 2006.

[34] O. Sidorenkov, O. Nilssen, T. Brenn, S. Martiushov, V. L. Arkhipovsky, and A. M. Grjibovski, "Prevalence of the metabolic syndrome and its components in Northwest Russia: the Arkhangelsk study," BMC Public Health, vol. 10, no. 1, p. 23, 2010.

[35] D. L. Jacobson, S. J. Gange, N. R. Rose, and N. M. H. Graham, "Epidemiology and estimated population burden of selected autoimmune diseases in the United States," Clinical Immunology and Immunopathology, vol. 84, no. 3, pp. 223-243, 1997.

[36] L. Sieminska, C. Wojciechowska, K. Walczak et al., "Associations between metabolic syndrome, serum thyrotropin, and thyroid antibodies status in postmenopausal women, and the role of interleukin-6," Endokrynologia Polska, vol. 66, no. 5, pp. 394-403, 2015.

[37] K. Agbaht, Y. Mercan, S. Kutlu, M. F. Alpdemir, and T. Sezgin, "Obesity with and without metabolic syndrome: do vitamin D and thyroid autoimmunity have a role?," Diabetes Research and Clinical Practice, vol. 106, no. 1, pp. 27-34, 2014.

[38] D. Fairweather, S. Frisancho-Kiss, and N. R. Rose, "Sex differences in autoimmune disease from a pathological perspective," The American Journal of Pathology, vol. 173, no. 3, pp. 600609, 2008.

[39] M. Cutolo, P. Montagna, R. Brizzolara et al., "Sex hormones modulate the effects of Leflunomide on cytokine production by cultures of differentiated monocyte/macrophages and synovial macrophages from rheumatoid arthritis patients," Journal of Autoimmunity, vol. 32, no. 3-4, pp. 254-260, 2009.

[40] A. Lleo, P. M. Battezzati, C. Selmi, M. E. Gershwin, and M. Podda, "Is autoimmunity a matter of sex?," Autoimmunity Reviews, vol. 7, no. 8, pp. 626-630, 2008.
[41] L. C. Golden and R. Voskuhl, "The importance of studying sex differences in disease: the example of multiple sclerosis," Journal of Neuroscience Research, vol. 95, no. 1-2, pp. 633-643, 2017.

[42] N. Wang, L. Kuang, B. Han et al., "Follicle-stimulating hormone associates with prediabetes and diabetes in postmenopausal women," Acta Diabetologica, vol. 53, no. 2, pp. 227-236, 2016. 


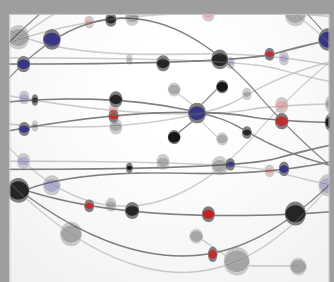

The Scientific World Journal
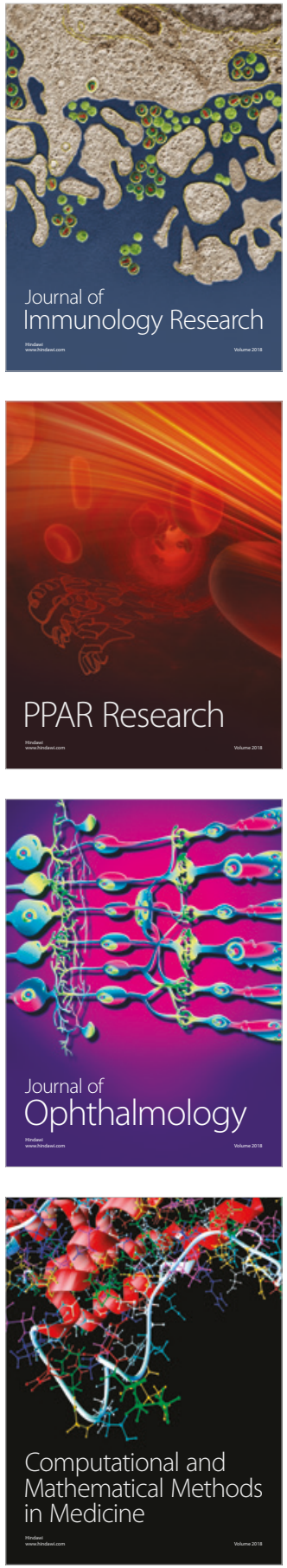

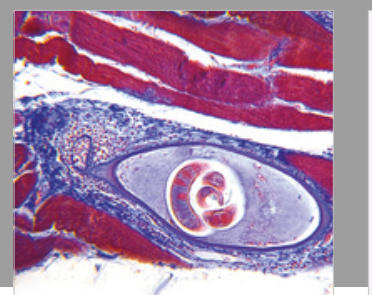

Gastroenterology Research and Practice

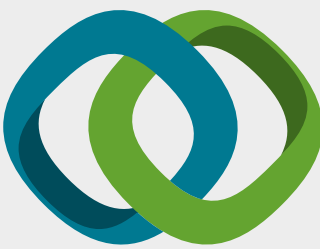

\section{Hindawi}

Submit your manuscripts at

www.hindawi.com
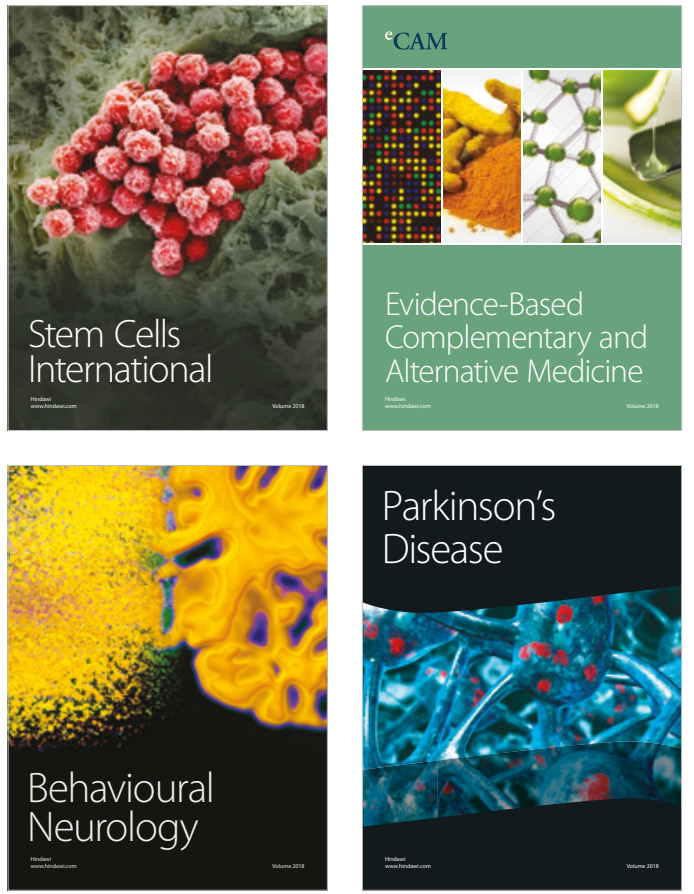

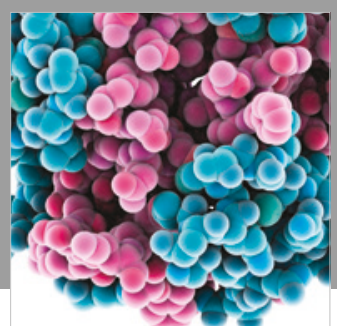

ournal of

Diabetes Research

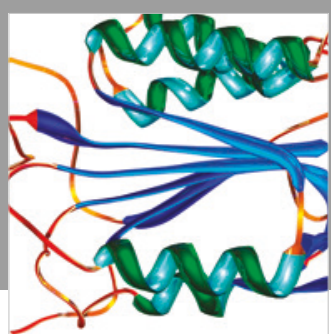

Disease Markers
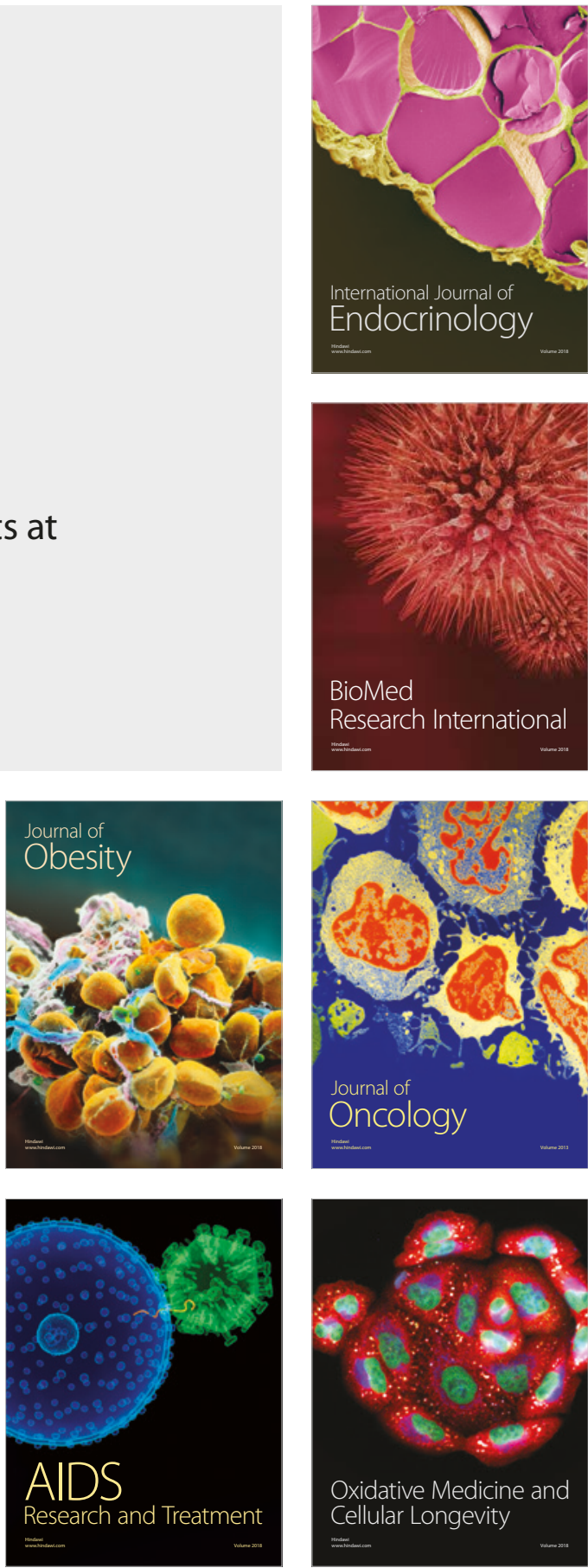